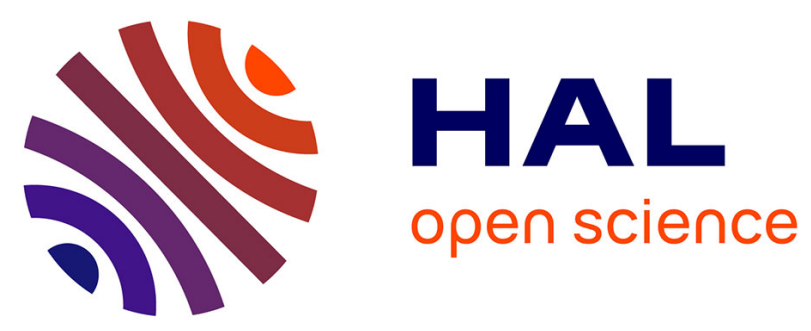

\title{
De Novo Truncating Mutations in the kinetochore-microtubules attachment gene CHAMP1 Cause Syndromic Intellectual Disability
}

Bertrand Isidor, Sébastien Küry, Jill A. Rosenfeld, Thomas Besnard, Sébastien Schmitt, Shelagh Joss, Sally J. Davies, Robert Roger Lebel, Alex Henderson, Christian P. Schaaf, et al.

\section{To cite this version:}

Bertrand Isidor, Sébastien Küry, Jill A. Rosenfeld, Thomas Besnard, Sébastien Schmitt, et al.. De Novo Truncating Mutations in the kinetochore-microtubules attachment gene CHAMP1 Cause Syndromic Intellectual Disability. Human Mutation, 2016, 37 (4), pp.354-358. 10.1002/humu.22952 . hal-01259225

HAL Id: hal-01259225

https://hal-univ-rennes1.archives-ouvertes.fr/hal-01259225

Submitted on 26 Sep 2016

HAL is a multi-disciplinary open access archive for the deposit and dissemination of scientific research documents, whether they are published or not. The documents may come from teaching and research institutions in France or abroad, or from public or private research centers.
L'archive ouverte pluridisciplinaire HAL, est destinée au dépôt et à la diffusion de documents scientifiques de niveau recherche, publiés ou non, émanant des établissements d'enseignement et de recherche français ou étrangers, des laboratoires publics ou privés. 
Humu-2015-0435

Brief Report

De Novo Truncating Mutations in the kinetochore-microtubules attachment gene CHAMP1 Cause Syndromic Intellectual Disability

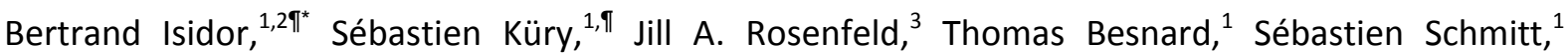
Shelagh Joss, ${ }^{4}$ Sally J Davies, ${ }^{5}$ Robert Roger Lebel, ${ }^{6}$ Alex Henderson, ${ }^{7}$ Christian P. Schaaf, ${ }^{3}$ Haley E. Streff, ${ }^{3}$ Yaping Yang, ${ }^{3}$ Vani Jain, ${ }^{5}$ Nodoka Chida, ${ }^{8}$ Xenia Latypova, ${ }^{1}$ Cédric Le Caignec, ${ }^{1,2}$ Benjamin Cogné, ${ }^{1}$ Sandra Mercier, ${ }^{1}$ Marie Vincent, ${ }^{1}$ Estelle Colin, ${ }^{9}$ Dominique Bonneau, ${ }^{9}$ Anne-Sophie Denommé, ${ }^{9}$ Philippe Parent, ${ }^{10}$ Brigitte Gilbert-Dussardier, ${ }^{11}$ Sylvie Odent, ${ }^{12}$ Annick Toutain, ${ }^{13}$ Amélie Piton, ${ }^{14}$ Christian Dina, ${ }^{15,16}$ Audrey Donnart, ${ }^{15,16}$ Pierre Lindenbaum, ${ }^{15,16}$ Eric Charpentier, ${ }^{15,16}$ Richard Redon, ${ }^{15,16}$ Kenji lemura, ${ }^{8}$ Masanori Ikeda, ${ }^{8}$ Kozo Tanaka, ${ }^{8, \&}$ Stéphane Bézieau ${ }^{1, \&}$

${ }^{1}$ CHU Nantes, Service de Génétique Médicale, 9 quai Moncousu, 44093 Nantes CEDEX 1, France

${ }^{2}$ INSERM, UMR-S 957, 1 Rue Gaston Veil, 44035 Nantes, France

${ }^{3}$ Department of Molecular and Human Genetics, Baylor College of Medicine, Houston, Texas, USA

${ }^{4}$ Department of Clinical Genetics, NHS Greater Glasgow and Clyde, Glasgow, United Kingdom 
${ }^{5}$ All Wales Medical Genetic Service, University Hospital of Wales, Cardiff.

${ }^{6}$ Genetics Section, Department of Pediatrics, SUNY Upstate Medical University, Syracuse, NY, USA

${ }^{7}$ Northern Genetics Service, Newcastle upon Tyne Hospitals NHS Foundation Trust, Newcastle upon Tyne NE1 3BZ, UK

8 Department of Molecular Oncology, Institute of Development, Aging and Cancer, Tohoku University, Sendai, Japan

${ }^{9} \mathrm{CHU}$ Angers, Département de Biochimie et Génétique, 4 rue Larrey, 49100 Angers, France et UMR INSERM 1083 - CNRS 6214

${ }^{10}$ CHRU Brest, Génétique médicale, 29609 Brest, France

${ }^{11}$ CHU Poitiers, Service de Génétique, BP577, 86021 Poitiers, France; EA 3808 Université Poitiers, France

${ }^{12}$ CHU Rennes, Service de Génétique Clinique, CNRS UMR6290, Université Rennes1, Rennes, France

${ }^{13} \mathrm{CHU}$ Tours, Service de Génétique, 2 Boulevard Tonnellé, 37044 Tours, France

${ }^{14}$ Département Médecine translationelle et neurogénétique, Institut de Génétique et de Biologie Moléculaire et Cellulaire (IGBMC), Institut National de Santé et de Recherche Médicale (INSERM) U964/Centre National de Recherche Scientifique (CNRS) UMR 7104/Université de Strasbourg, 67404 Illkirch, France, Laboratoire de diagnostic génétique, Hôpitaux Universitaires de Strasbourg, 67000 Strasbourg, France

${ }^{15}$ Inserm, UMR 1087, l'institut du thorax, CHU Nantes, Nantes, France 
${ }^{16}$ CNRS, UMR 6291, Université de Nantes, Nantes, France

"These authors contributed equally to this work

\& These authors contributed equally to this work

* Corresponding Author: Dr Bertrand Isidor, Service de Génétique Médicale, Centre Hospitalier Universitaire de Nantes 1, place Alexis Ricordeau, 44093 NANTES Cedex 01, France. Tel: +33 14008 35 41. Fax: +33 2400876 47. E-mail: bertrand.isidor@chu-nantes.fr

Grant sponsors: French Ministry of Health and Health Regional Agency from Poitou-Charentes (HUGODIMS, 2013, RC14_0107) 


\section{Abstract}

A rare syndromic form of intellectual disability with impaired speech was recently found associated with mutations in CHAMP1 (chromosome alignment-maintaining phosphoprotein 1), the protein product of which is directly involved in microtubule-kinetochore attachment. Through whole-exome sequencing in six unrelated non-consanguineous families having a sporadic case of intellectual disability, we identified six novel de novo truncating mutations in CHAMP1: c.1880C>G p.(Ser627*), c.1489C>T; $\quad$ p.(Arg497*), c.1876_1877delAG; $\quad$ p.(Ser626Leufs*4), $\quad$ c.1043G>A; p.(Trp348*), c.1002G>A; p.(Trp334*) and c.958_959delCC; p.(Pro320*). Our clinical observations confirm the phenotypic homogeneity of the syndrome, which represents therefore a distinct clinical entity. Besides, our functional studies show that CHAMP1 protein variants are delocalized from chromatin and are unable to bind to two of its direct partners, POGZ and HP1. These data suggest a pathogenic mechanism of the CHAMP1-associated intellectual disability syndrome mediated by direct interacting partners of CHAMP1, several of which are involved in chromo/kinetochore-related disorders.

Key Words: Intellectual disability, CHAMP1, microcephaly, POGZ, HP1, kinetochores, microtubules 
Division of neural progenitor cells is one of the key processes, which, together with neuronal polarization and differentiation, shapes the complex architecture of the developing human brain [Sakakibara, et al., 2013]. Microtubules play an important part in these different morphogenetic processes, and notably in cell division, where they contribute to the partition of chromosomes in daughter cells by empowering chromosome movements and by linking chromosomes to spindles through binding to kinetochores [Duro and Marston, 2015]. Chromosome segregation accuracy is highly dependent on attachment of sister kinetochores to microtubules from opposite spindle poles [Tanaka and Watanabe, 2008]. Such a phenomenon requires the coordinated action of numerous proteins acting directly on the microtubule-binding interface of kinetochores [Itoh, et al., 2011], correcting improper microtubule-kinetochore attachments [Ruchaud, et al., 2007], and controlling the quality of these attachments [Musacchio and Salmon, 2007]. Mutations in microtubule-related genes, including KIF5C, KIF2A, DYNC1H1 and TUBG1, have been shown to cause malformations of cortical development associated with severe intellectual disability (ID) and/or microcephaly [Poirier, et al., 2013].

Following a first finding from a large cohort study [Rauch, et al., 2012], Hempel et al. [Hempel, et al., 2015] documented recently five ID cases with severe impaired speech associated with mutations in CHAMP1 (chromosome alignment-maintaining phosphoprotein 1; MIM\# 616327), the last subtelomeric gene from the long arm of chromosome 13 (chr13:114,314,490-114,327,328, GRCh37/hg19). CHAMP1 contains two untranslated exons and one translated exon encoding an 812amino-acid zinc-finger protein, which is directly involved in microtubule-kinetochore attachment. 
CHAMP1 protein product is highly conserved across vertebrates and contains three characteristic repeat motifs named WK, SPE, and FPE [Itoh, et al., 2011].

Through a multicenter collaborative study on sporadic cases with ID, we were able to identify six new de novo variants very likely affecting the function of CHAMP1 in children with ID. The six affected children presented here were enrolled in three different programs or centers investigating the molecular basis of intellectual disabilities (Table 1): the Western France consortium HUGODIMS (French acronym standing for "Projet inter-régional Français des Hôpitaux Universitaires du Grand Ouest pour l'exploration par approche exome des causes moléculaires de Déficience Intellectuelle isolée ou syndromique Modérée à Sévère"; proband F1-II.1), the Wellcome Trust Sanger Institute British program Deciphering Developmental Disorders (DDD; patients F2-II.1, F5-II.1 and F6-II.1), and the American Baylor Miraca Genetics Laboratory (BMGL) clinical exome testing cohort (patients F3-II.1 and F4-II.1). Regarding both HUGODIMS and DDD, probands were included together with their biological parents, following a trio strategy, whereas the diagnostics protocol defined by the BMGL required sampling of the patient only and testing of parents for variants of interest. Written informed consent for inclusion in the study and consent for the publication of photographs was obtained for all patients. The study has been approved by the CHU de Nantesethics committee (number CCTIRS: 14.556). Clinical evaluation was performed for each patient by at least one expert clinical geneticist; it revealed very similar phenotypic features shared by all six affected individuals: severe or moderate ID, hypotonia, absent or very poor speech, microcephaly (3/6) ranging from -2 SD to -4 SD, and facial dysmorphy (Fig. 1, Table 1, Supp. Methods and Supp. Table S1). For all patients, brain MRIs were considered normal or with nonspecific findings. Routine clinical genetic and metabolic screenings were negative. Detailed observations are reported in Supp. Methods and Supp. Table S1. 
By applying a whole-exome sequencing (WES) strategy to the first trio (Family 1), we identified the de novo nonsense heterozygous variant NM_032436.2:c.1880C>G; p.(Ser627*) in CHAMP1 in patient F1-II.1 (Supp. Fig. S1), which was verified by Sanger sequencing (Supp. Fig. S2). We did not identify any X-chromosomal, compound heterozygous or homozygous candidate variants in known ID genes. From the BMGL independent cohort of 5300 individuals undergoing clinical WES, we identified two additional patients (patients F3-II.1 and F4-II.1) having a truncating variant in CHAMP1 (NM_032436.2:c.1876_1877delAG; p.(Ser626Leufs*4) and c.1043G>A; p.(Trp348*)). We validated these variants by Sanger sequencing, and parental genotyping confirmed their de novo origin (Supp. Fig. S2). Three further patients recruited from the DDD cohort exhibited a de novo nonsense CHAMP1 variant verified by capillary sequencing (Supp. Fig. S2): c.1002G>A (p.(Trp334*)) in patient F2-II.1, c.958_959delCC (p.(Pro320*)) in patient F5-II.1 and c.1489C>T (p.(Arg497*)) in patient F6-II.1. None of the six mutations was present either in over 6,500 in-house exomes or in any public variant databases, save c.1489C $>$ T (p. $\left(\operatorname{Arg} 497^{*}\right)$ which is referenced in dbSNP and ClinVar (rs782397980;RCV000191999.2). Two of these patients (patients F2-II.1 and F6-II.1) were previously reported without clinical description as part of a large cohort study on ID [Deciphering Developmental Disorders, 2015].

Our six patients show a phenotype very similar to the patients reported by Hempel et al. who already noted phenotypic similarities with cases of subtelomeric 13q deletions reported in copy number variation databases [Hempel, et al., 2015]. The syndromic form of ID due to CHAMP1 mutations might be difficult to recognize clinically, but association of neonatal hypotonia with motor delay, severe speech impairment, facial anomalies (round face, facial hypotonia, hypertelorism) and microcephaly should lead to evoke this diagnosis. 
CHAMP1 expression is consistent with a role in ID. In mice, proteins levels are detected in a few organs only, including brain, and also thymus, testis, and ovary (Supp. Fig. S3). In situ hybridization with a probe to mouse Champ1 reported in EMAGE database showed expression in the thymus, gonads and developing brain at embryonic day (E) 14.5. Expression was seen in the ventricular zone and the cortical plate, hereby consistent with a putative role in progenitor cells [Richardson, et al., 2014]. All de novo mutations identified in our patients and in the five ones reported by Hempel et al. (2015) create a premature stop codon located in the only coding exon of CHAMP1, exon $3 \mathrm{~b}$, and affect residues highly conserved across species. Interestingly, the resulting truncated proteins all lack the C-terminal region containing three zinc-finger domains (Supp. Fig. S1), which region is crucial for CHAMP1 localization to mitotic chromatin [Itoh, et al., 2011].

To assess a possible effect of the variants found in our patients, we examined subcellular localization of three of the CHAMP1 protein variants, derived from c.1880C $>\mathrm{G}$, c.1043G $>A$, c.1876_1877delAG , by expressing them as Green Fluorescent Protein (GFP)-fusion proteins in HeLa cells (Supp. Fig. S4A). We found that, unlike full-length CHAMP1, they do not localize to chromatin in metaphase cells (Supp. Fig. S4B). In interphase cells, full-length CHAMP1 localizes to heterochromatic regions densely stained with DAPI (Supp. Fig. S4B), probably reflecting the interaction with HP1 (heterochromatin protein 1), which is involved in heterochromatin formation.

In contrast, two CHAMP1 mutants (c.1880C>G; p.(Ser627*), and c.1876_1877delAG; p.(Ser626Leufs*4)) showed diffuse nuclear localization (Supp. Fig. S4B). Another CHAMP1 mutant (c.1043G>A; p.(Trp348*)), which further lacks the middle region containing the FPE and WK motifs (Supp. Fig. S1), localized to the cytoplasm (Supp. Fig. S4), suggesting that this middle region contains a nuclear localization signal. These data suggest that these CHAMP1 truncated mutants cannot localize to chromatin. 
To further verify the defects in the CHAMP1 mutants, we investigated the interaction of CHAMP1 with other proteins. CHAMP1 was identified as an interacting protein with Mad2L2 (Mitotic arrest-deficient 2, S.Cerevisiae, Homolog-like-2), which binds to the middle region of CHAMP1 containing the WK motifs [Itoh, et al., 2011]. In addition, HP1 (Heterochromatin protein 1) and POGZ (POGO transposable element with ZNF domain) were found to interact with CHAMP1 by proteomic analysis (K.T., unpublished data), which was confirmed by western blotting analysis (Supp. Fig. S5A). POGZ (pogo transposable element-derived protein with zinc finger domain) was found as an HP1binding protein together with CHAMP1 [Nozawa, et al., 2010]. CHAMP1, Mad2L2, HP1, and POGZ were also identified as a complex bound to trimethylated histone H3K9 [Vermeulen, et al., 2010]. We determined the interaction domains of CHAMP1 with HP1 and POGZ by in vitro binding assays using purified proteins (Supp. Fig. S5B). HP1 was found to bind to the C-terminal fragment of CHAMP1, but not the C-terminal fragment lacking the zinc-finger domains (Supp. Fig. S5C), indicating that HP1 binds to the C-terminal region containing the zinc-finger domains. We also found that the C-terminal fragment of POGZ interacts with the same C-terminal region of CHAMP1 containing the zinc-finger domains (Supp. Fig. S5D). As this region is lacking in all CHAMP1 mutants, these mutants are probably incapable of binding to both HP1 and POGZ. The six mutations observed in the patients lie in regions predicted to be disordered (Supp. Fig. S6) and are located between domains at both termini which would contain two and three zinc-finger respectively, according to three-dimensional modeling of CHAMP1 protein product (Supp. Fig. S7); the six mutations give all rise to truncation of CHAMP1, removing the three zinc-fingers domain at the C-terminus (Supp. Fig. S7).

Overall, our functional studies show that truncation protein variants of CHAMP1 are likely delocalized from chromatin and unable to bind to two of its direct partners, POGZ and HP1. These 
data are not sufficient to fully understand the pathogenic mechanism of the CHAMP1-associated ID syndrome, but they allow us to formulate several hypotheses.

First, pathogenicity may result from loss of mitotic function of CHAMP1. This protein is involved in kinetochore-microtubule attachment in a way that maintains chromosome alignment on the metaphase plate [Itoh, et al., 2011]. Therefore, it is plausible that chromosome segregation defects in neuronal progenitor cells may cause this developmental disorder.

Alternatively, the pathogenic mechanism leading to ID may result from impaired CHAMP1 binding to its partners; it was reported indeed that disruption of POGZ also causes mitotic defects, abnormal chromosome segregation, nuclear fragmentation, and disrupted mitotic HP1-alpha localization [Nozawa, et al., 2010]. It is intriguing that de novo loss-of-function mutations in POGZ have recently been identified in individuals affected by neurodevelopmental disorders with a similar phenotype (ID, microcephaly) [Fromer, et al., 2014; lossifov, et al., 2012; Neale, et al., 2012]. Our data show that all the CHAMP1 mutants lack the C-terminal region which is required for interaction with POGZ, suggesting that the inability to form complexes with POGZ may affect the CHAMP1 function. Furthermore, as for CHAMP1, heterozygous de novo POGZ mutations found in patients with ID give rise to truncations of the C-terminal region, which is required for interaction with CHAMP1 (Supp. Fig. S5D).

While CHAMP1 may be haploinsufficient, with loss of function of a single copy of the gene sufficiently impairs function to cause disease, dominant-negative or gain-of-function effects of the mutations observed on CHAMP1 protein activity cannot be ruled out. All the nonsense or frameshift mutations identified are located in the only coding exon of the gene and they might therefore escape nonsense-mediated mRNA decay (NMD). In such case, the mutated mRNAs would be 
expected to be stable and the resulting truncated proteins functional. This hypothesis is corroborated by the normal phenotype of mice heterozygous for Champ1 deletions (K.I. and K.T., unpublished data). On the other hand, mice homozygous for the same deletions die soon after birth (K.I. and K.T., unpublished data). The reason for the death is under investigation, but restricted expression of Champ1 in mice including brain (Supp. Fig. S3) implies death could be secondary to neurological impairment. Interestingly, HP1 $\beta-/-$ mice also die in the neonatal period with abnormal cerebral cortex development and reduced proliferation of neuronal precursors [Aucott, et al., 2008].

In conclusion, our clinical and experimental observations confirm the phenotypic homogeneity of the rare ID syndrome described by Hempel et al. and caused by de novo truncating mutations in CHAMP1 [Hempel, et al., 2015]. These findings expand the phenotypic spectrum of chromo/kinetochore disorders, several of which are caused by mutations in direct interacting partners of CHAMP1. The identification of CHAMP1 as a novel ID gene will increase the diagnostic yield of exome or genome sequencing in individuals with severe ID, and our data suggest that deciphering the function of CHAMP1 partners would very likely help to elucidate the pathophysiology of some neurodevelopmental disorders.

The reported sequence variants NM_032436.2:c.1880C>G, p.(Ser627*); c.1002G>A, p.(Trp334*); c.1876_1877delAG, p.(Ser626Leufs*4); c.1043G>A， (p.(Trp348*); c.958_959delCC, p.(Pro320*); and c.1489C>T, ( $\mathrm{p} .\left(\operatorname{Arg} 497^{*}\right)$ have been deposited in the Leiden Open Variation Database at http://www.lovd.nl/CHAMP1 under accession numbers 0000077846, 0000077847, $0000077848,0000077981,0000077984$, and 0000077985. 


\section{Acknowledgments}

We would like to thank all families for participating in this study. We acknowledge HUGODIMS consortium. We thank Frédérique Allaire from the Health Regional Agency of PoitouCharentes for supporting this project. We thank Léa Ferrand, Emilie Le Blanc for grant and data management. We are most grateful to the genomics and bioinformatics core facilities of Nantes (Biogenouest) for their technical support. This work was supported by a Grant-in-Aid for Scientific Research from the Japanese Society of Promotion of Science; a Grant-in-Aid for Scientific Research from the Ministry of Education Culture, Sports, Science and Technology of Japan; a grant from the Project for Development of Innovative Research on Cancer Therapeutics (P-Direct), The Japan Agency for Medical Research and Development; and a grant from Takeda Science Foundation. Dr. Schaaf's work is generously supported by the Joan and Stanford Alexander Family. We thank the American Baylor Miraca Genetics Laboratory (BMGL) for its very precious contribution to the present work. We also acknowledge the Deciphering Developmental Disorders (DDD) Study, and more especially Dr. Matthew Hurles, who, on behalf of DDD, fairly and kindly allowed us to use and present the results of whole-exome sequencing for patients F2-II.1, F5-II.1 and F6-II.1. We acknowledge also the DECIPHER Consortium which contributed to the exchange of genetic and clinical data between the teams. The authors would like to thank the Exome Aggregation Consortium and the groups that provided exome variant data for comparison. A full list of contributing groups can be found at http://exac. broadinstitute.org/about.

The funders had no role in study design, data collection and analysis, decision to publish, or preparation of the manuscript. 


\section{References}

Aucott R, Bullwinkel J, Yu Y, Shi W, Billur M, Brown JP, Menzel U, Kioussis D, Wang G, Reisert I, Weimer J, Pandita RK et al. 2008. HP1-beta is required for development of the cerebral neocortex and neuromuscular junctions. J Cell Biol 183(4):597-606.

Deciphering Developmental Disorders S. 2015. Large-scale discovery of novel genetic causes of developmental disorders. Nature 519(7542):223-8.

Duro E, Marston AL. 2015. From equator to pole: splitting chromosomes in mitosis and meiosis. Genes Dev 29(2):109-22.

Fromer M, Pocklington AJ, Kavanagh DH, Williams HJ, Dwyer S, Gormley P, Georgieva L, Rees E, Palta P, Ruderfer DM, Carrera N, Humphreys I et al. 2014. De novo mutations in schizophrenia implicate synaptic networks. Nature 506(7487):179-84.

Hempel M, Cremer K, Ockeloen CW, Lichtenbelt KD, Herkert JC, Denecke J, Haack TB, Zink AM, Becker J, Wohlleber E, Johannsen J, Alhaddad B et al. 2015. De Novo Mutations in CHAMP1 Cause Intellectual Disability with Severe Speech Impairment. Am J Hum Genet 97(3):493500.

Iossifov I, Ronemus M, Levy D, Wang Z, Hakker I, Rosenbaum J, Yamrom B, Lee YH, Narzisi G, Leotta A, Kendall J, Grabowska E et al. 2012. De novo gene disruptions in children on the autistic spectrum. Neuron 74(2):285-99. 
Itoh G, Kanno S, Uchida KS, Chiba S, Sugino S, Watanabe K, Mizuno K, Yasui A, Hirota T, Tanaka K. 2011. CAMP (C13orf8, ZNF828) is a novel regulator of kinetochore-microtubule attachment. EMBO J 30(1):130-44.

Musacchio A, Salmon ED. 2007. The spindle-assembly checkpoint in space and time. Nat Rev Mol Cell Biol 8(5):379-93.

Neale BM, Kou Y, Liu L, Ma'ayan A, Samocha KE, Sabo A, Lin CF, Stevens C, Wang LS, Makarov V, Polak P, Yoon S et al. 2012. Patterns and rates of exonic de novo mutations in autism spectrum disorders. Nature 485(7397):242-5.

Nozawa RS, Nagao K, Masuda HT, Iwasaki O, Hirota T, Nozaki N, Kimura H, Obuse C. 2010. Human POGZ modulates dissociation of HP1alpha from mitotic chromosome arms through Aurora B activation. Nat Cell Biol 12(7):719-27.

Poirier K, Lebrun N, Broix L, Tian G, Saillour Y, Boscheron C, Parrini E, Valence S, Pierre BS, Oger M, Lacombe D, Genevieve D et al. 2013. Mutations in TUBG1, DYNC1H1, KIF5C and KIF2A cause malformations of cortical development and microcephaly. Nat Genet 45(6):639-47.

Rauch A, Wieczorek D, Graf E, Wieland T, Endele S, Schwarzmayr T, Albrecht B, Bartholdi D, Beygo J, Di Donato N, Dufke A, Cremer K et al. 2012. Range of genetic mutations associated with severe non-syndromic sporadic intellectual disability: an exome sequencing study. Lancet 380(9854):1674-82.

Richardson L, Venkataraman S, Stevenson P, Yang Y, Moss J, Graham L, Burton N, Hill B, Rao J, Baldock RA, Armit C. 2014. EMAGE mouse embryo spatial gene expression database: 2014 update. Nucleic Acids Res 42(Database issue):D835-44. 
Ruchaud S, Carmena M, Earnshaw WC. 2007. The chromosomal passenger complex: one for all and all for one. Cell 131(2):230-1.

Sakakibara A, Ando R, Sapir T, Tanaka T. 2013. Microtubule dynamics in neuronal morphogenesis. Open Biol 3(7):130061.

Tanaka K, Watanabe Y. 2008. Chromatid cohesion: acetylation joins the sisters. Curr Biol 18(19):R917-9.

Vermeulen M, Eberl HC, Matarese F, Marks H, Denissov S, Butter F, Lee KK, Olsen JV, Hyman AA, Stunnenberg HG, Mann M. 2010. Quantitative interaction proteomics and genome-wide profiling of epigenetic histone marks and their readers. Cell 142(6):967-80.

\section{Figure legend}

Figure 1. Frontal facial photographs of the six patients with CHAMP1 de novo variants included in the study. a: Patient F1-II.1, b: Patient F2-II.1, c: Patient F3-II.1, d: Patient F4-II.1, e: Patient F5-II.1, and f: Patient F6-II.1. Note the clinical similarities, including a round face, hypertelorism, and a large mouth. 
Table 1. Detailed clinical features of patients with CHAMP1 mutation

\begin{tabular}{|c|c|c|c|c|c|c|}
\hline & $\begin{array}{l}\text { Patient F1-II.1 } \\
\text { (France) }\end{array}$ & $\begin{array}{l}\text { Patient F2-II.1 } \\
\text { (UK) }\end{array}$ & $\begin{array}{l}\text { Patient F3-II.1 } \\
\text { (USA) }\end{array}$ & $\begin{array}{l}\text { Patient F4-II.1 } \\
\text { (USA) }\end{array}$ & $\begin{array}{l}\text { Patient F5-II.1 } \\
\text { (UK) }\end{array}$ & $\begin{array}{l}\text { Patient } \\
\text { (UK) }\end{array}$ \\
\hline Gender & Male & Male & Female & Male & Female & Female \\
\hline $\begin{array}{l}\text { Developmental } \\
\text { delay/intellectual } \\
\text { disability }\end{array}$ & + (severe) & + (severe) & + (severe) & + (severe) & + (moderate) & + (seve \\
\hline Failure to thrive & + & - & - & + & - & - \\
\hline Delay in walking & + & + & + & + & + & + \\
\hline Speech delay & + & + & + & + & + & + \\
\hline Speech abilities & none & none & very limited & none & very limited & very lin \\
\hline $\begin{array}{l}\text { Behavioural } \\
\text { anomalies }\end{array}$ & - & - & + & + & + & - \\
\hline Seizures & - & - & + & - & - & ND \\
\hline $\begin{array}{l}\text { Skeletal } \\
\text { abnormalities }\end{array}$ & + & + & + & + & + & - \\
\hline Facial dysmorphism & + & - & + & + & + & + \\
\hline Eye anomalies & + & + & + & + & ND & + \\
\hline Feeding difficulties & + & - & ND & + & + & ND \\
\hline $\begin{array}{l}\text { CHAMP1 mutation } \\
\text { (NM_032436.2)* }\end{array}$ & $\begin{array}{l}\text { c. } 1880 C>G, \\
\text { p. }(\text { Ser627*) }\end{array}$ & $\begin{array}{l}\text { c. } 1002 \mathrm{G}>\mathrm{A}, \\
\text { p. }\left(\operatorname{Trp} 334^{*}\right)\end{array}$ & $\begin{array}{l}\text { c.1876_1877delAG; } \\
\text { p.(Ser626Leufs*4) }\end{array}$ & $\begin{array}{l}\text { c. } 1043 \mathrm{G}>\mathrm{A}, \\
\text { p. }\left(\operatorname{Trp} 348^{*}\right)\end{array}$ & $\begin{array}{l}\text { c.958_959delCC, } \\
\text { p.(Pro320*) }\end{array}$ & $\begin{array}{l}\text { c. } 1489 \\
\text { p.(Arg4 }\end{array}$ \\
\hline
\end{tabular}


* Nomenclature HGVS V2.0 according to mRNA reference sequence NM_032436.2. Nucleotide numbering uses +1 as the $A$ of the ATG translation initiation codon in the reference sequence, with the initiation codon as codon 1.

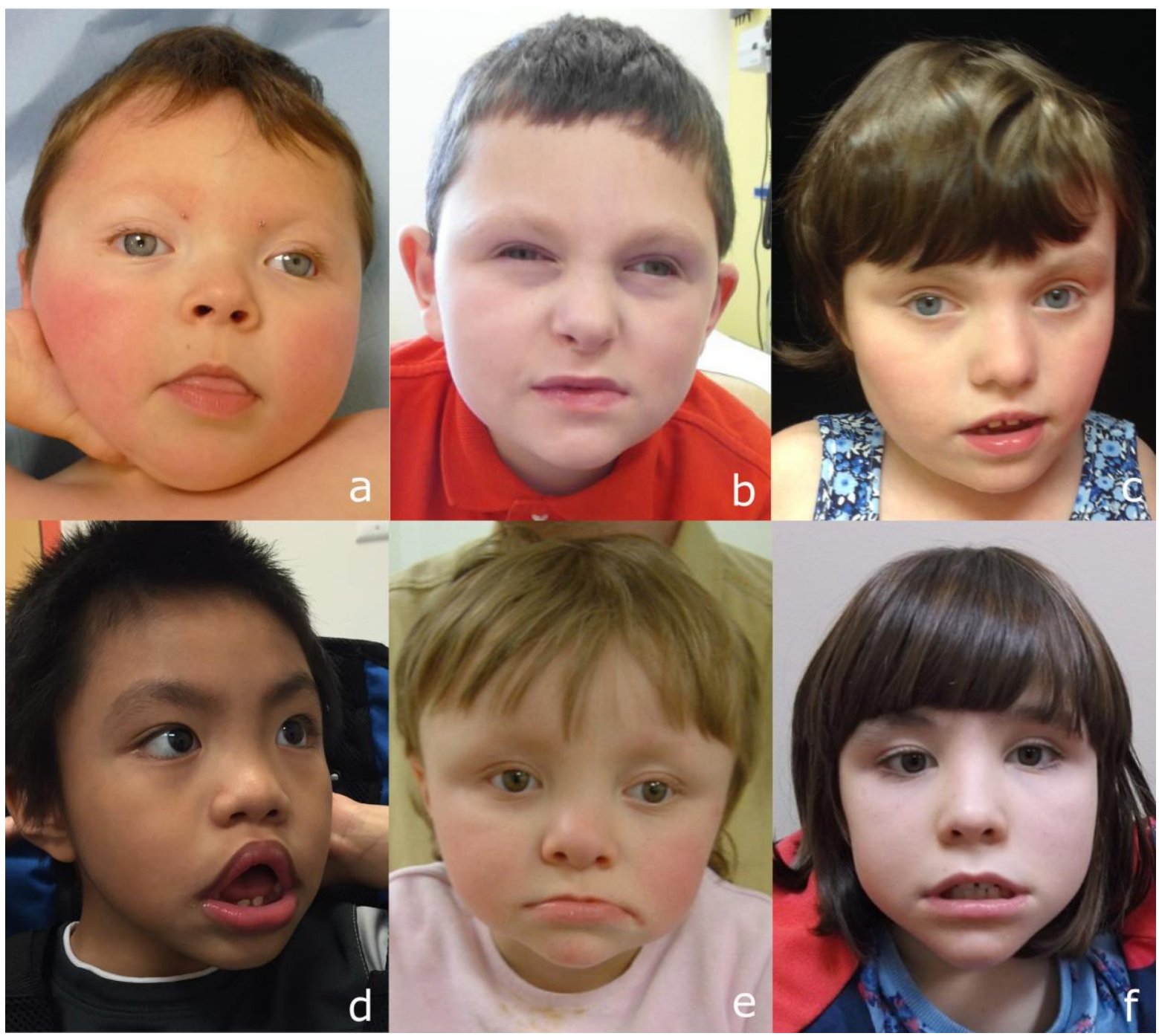

